10-20-1997

\title{
Fiber-Optics Surface-Light-Scattering Spectrometer
}

Padetha Tin

\section{J. Adin Mann}

William V. Meyer

Thomas W. Taylor

Cleveland State University, t.taylor@csuohio.edu

Follow this and additional works at: https://engagedscholarship.csuohio.edu/sciphysics_facpub

Part of the Physics Commons

How does access to this work benefit you? Let us know!

\section{Publisher's Statement}

This paper was published in Applied Optics and is made available as an electronic reprint with the permission of OSA. The paper can be found at the following URL on the OSA website: http://www.opticsinfobase.org/ao/abstract.cfm?URI=ao-36-30-7601. Systematic or multiple reproduction or distribution to multiple locations via electronic or other means is prohibited and is subject to penalties under law.

\section{Original Citation}

Tin, Padetha, J. Adin Mann, William V. Meyer, and Thomas W. Taylor. "Fiber-Optics Surface-LightScattering Spectrometer." Applied Optics 36 (1997): 7601-7604.

\section{Repository Citation}

Tin, Padetha; Mann, J. Adin; Meyer, William V.; and Taylor, Thomas W., "Fiber-Optics Surface-Light-Scattering Spectrometer" (1997). Physics Faculty Publications. 108.

https://engagedscholarship.csuohio.edu/sciphysics_facpub/108

This Article is brought to you for free and open access by the Physics Department at EngagedScholarship@CSU. It has been accepted for inclusion in Physics Faculty Publications by an authorized administrator of EngagedScholarship@CSU. For more information, please contact library.es@csuohio.edu. 


\title{
Fiber-optics surface-light-scattering spectrometer
}

\author{
Padetha Tin, J. Adin Mann, Jr., William V. Meyer, and Thomas W. Taylor
}

\begin{abstract}
We have developed a fiber-optics surface-light-scattering spectrometer completely designed with optical fiber components. To the best of our knowledge, this is the first demonstration of a noninvasive measurement of the surface tension and the viscosity of simple liquid-vapor interfaces with a fiberoptics-based sensor system. With this approach we obtain a compact size, a significant increase in the signal-to-noise ratio, and the ability to select from a continuum of wave vectors. (C) 1997 Optical Society of America

Key words: Surface light scattering, surface tension, viscosity, fiber-optics homodyning.
\end{abstract}

\section{Introduction}

In order to determine surface tension and viscosity noninvasively, we measure the light scattered from the thermocapillary waves generated by thermally excited surface ripplons. This method was first introduced by Raman around 1925, and a brief history is given in Langevin's book. ${ }^{1}$ The method measures the power spectrum of a selection of the surface waves with wavelengths of the order of $0.1 \mathrm{~mm}$, corresponding to frequencies between approximately 5 and $50 \mathrm{kHz}$, depending on the material. The surface tension and viscosity are then deduced from the acquired autocorrelation function. The laser homodyne method is used in this technique by introduction of a local oscillator to beat against the scattered signal. In the earliest experiments, generation of the local oscillator was unstable, and the diffraction angle was selected by a narrow detector aperture. This resulted in low resolution, signal sensitivity that depended on the strength of the local oscillator, and poor reproducibility. The grating method is a substantial improvement over this early work and is well described in various publications; we discuss this next.

As interest in the characterization of thin films,

P. Tin and W. V. Meyer are with the Ohio Aerospace Institute, NASA Lewis Research Center, MS 105-1, 21000 Brookpark Road, Cleveland, Ohio 44135-3191. J. A. Mann, Jr. is with the Department of Chemical Engineering, Case Western Reserve University, Cleveland, Ohio 44106-7217. T. W. Taylor is with the Department of Physics, Cleveland State University, Cleveland, Ohio 44115-2440.

Received 28 March 1997; revised manuscript received 30 May 1997.

0003-6935/97/307601-04\$10.00/0

(C) 1997 Optical Society of America monolayers, and biological membranes grew, this noninvasive laser homodyne technique for measuring surface capillary waves and biological membranes progressively improved. Härd et al. developed a surface-light-scattering apparatus consisting of a grating placed in the optical beam path to create a constant local oscillator. ${ }^{2}$ The idea behind this was to project the image of the grating on a liquid-vapor interface and homodyne beat the scattered signal with the first-order diffracted beam at the photodetector. This approach provides a constant-value local oscillator for homodyning. It also selects the scattered $\mathbf{k}$ vector, which is an essential factor in the dispersion relation equation when one is deducing the surface tension and the viscosity (the scattered $\mathbf{k}$ vector can now be directly calculated from the grating constant). A detailed theory of surface-fluctuation spectroscopy for which the grating homodyne detection method is used has been discussed by Lading and co-workers ${ }^{3,4}$ and Langevin. ${ }^{1}$ They have analyzed the grating homodyne system, paying special attention to the corrections required in the data analysis algorithm that are due to the errors introduced by the instrument that could lead to systematic errors.

An alternative way to detect light scattering from thermal ripplon waves is to split a low-intensity beam from the main beam and combine it with the scattered light before it gets into the photodetector. Because the local-oscillator light and scattered light both originate from a common laser source whose output is split and then later recombined downstream, a delay line is inserted into one of the beam paths to compensate for any path differences. Mazur and Chung 5 used this type of spectrometer to measure surface properties of liquid-vapor interfaces. Sakai et al. also used this same technique to observe the ripplon propagation of liquid-vapor in- 


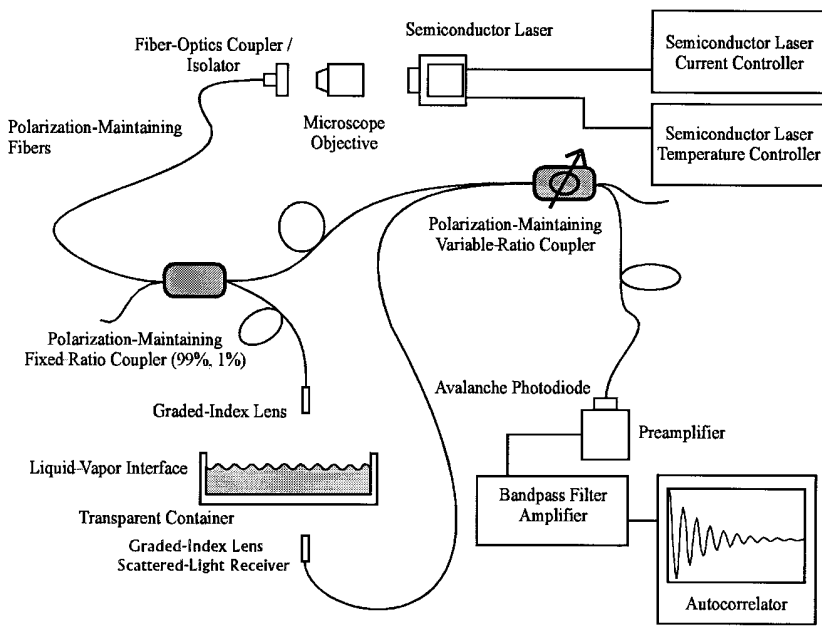

Fig. 1. FOSLSS transmission type.

terfaces at high wave numbers. By using the transmission diffraction method and some improvements of optics and electronics, they were able to extend the wave-numbers range to $4 \times 10^{4}-3 \times 10^{6}(10 \mathrm{kHz}-6$ $\mathrm{MHz}$ in the frequency range). ${ }^{6}$ Recently, Jørgensen showed that a conventional bulk-optics-type system can be used with an inexpensive multimode semiconductor laser diode, thus avoiding the onerous ion laser noises that can interfere with the ripplon. ${ }^{7}$ All these techniques use conventional bulk optics. Our system is based completely on fiber-optics technology. Thus one advantage of this system over the classical optical system described above is that the fiber-optics setup is compact and robust.

\section{Experimental Setup}

Figure 1 shows the optical setup of the fiber-optics surface-light-scattering spectrometer (FOSLSS). We chose a single-mode, semiconductor laser diode manufactured by Spectra Diode Labs, Model SDL 7412 H1S. The stripe structure of the active area was $1 \mu \mathrm{m} \times 3.5 \mu \mathrm{m}$ and it lased at a single longitudinal mode at $775 \mathrm{~nm}$. Maximum power output from this laser was $100 \mathrm{~mW}$ with no astigmatism correction. A 70\% coupling efficiency was obtained when the power output was launched into the polarization-maintaining single-mode optical fiber with a $20 \times$ microscope objective. To prevent mode hopping, the laser chip temperature was stabilized at $20^{\circ} \mathrm{C}$. We used a polarization-preserving fixedratio $(99.9 \%$ to $0.1 \%)$ coupler manufactured by Canadian Instrumentation and Research (Model 904) to split the laser power into two outputs. The higherpower fiber output $(99.9 \%)$ was launched onto the surface, and the other fiber output $(0.1 \%)$ was used as a local oscillator for homodyne beating. The higherpower beam was collimated with a 2-mm-diameter graded-index lens. The power output measured at the main fiber before the collimated light impinged on the liquid interface was $\sim 25 \mathrm{~mW}$.

The intensity of the light scattered from the liquidvapor interface is weak, ${ }^{1}$ and, because the surface acts as an optical lever arm in reflection, external vibrations make maintaining a mechanically stable interface quite difficult. Sakai et al. ${ }^{6}$ have shown that forward-scattering detection has two advantages over backscattering that help address these concerns. First, the forward-scattered light intensity is greater than the backward-scattered intensity. Second, and more importantly, the effective surface stability is $\sim 7$ times better when forward-scattering geometry is used instead of a traditional reflectiontype geometry. Therefore, for the initial tests of our instrument design, we tested the fiber-optics system with a collimated beam that traversed through the liquid-vapor interface under study. In this way we detected the scattered light exiting through (forward scattered by) the media. This setup requires a container with a high-quality polished transparent bottom. The gain in stability is dependent on the refractive index of the liquid media. Although forward scattering helps overcome low-frequency vibration and surface sloshing, one cannot use this setup for opaque liquids. An alternative technique to overcome surface sloshing for a bulk optics system is discussed in a separate paper in this feature issue. ${ }^{8}$

The scattered signal transmitted through the bottom of the container was picked up by another polarization-maintaining single-mode fiber by means of a graded-index lens. This scattered signal was combined with the local-oscillator light by a polarization-maintaining variable-ratio coupler $(\mathrm{Ca}-$ nadian Instrumentation and Research Model 905P). Homodyne beating was effectively achieved by controlling the amount of local-oscillator light with this variable-ratio coupler. Care must be taken to control stray light introduced by imperfect collimation of the light launched out of the graded-index lens. This dc stray light can introduce an additional constant local oscillator that prevents proper use of the local oscillator, which has been split with the fixedratio coupler to remix later with the scattered light. It is also quite important to prevent any backreflection from the bare fiber termination (the unused terminations in the fixed-ratio coupler, as well as those in the variable-ratio coupler). The signal was finally detected by a Hamamatsu C5460 avalanche photodiode. The scattered signal collected by fiber attached to the graded-index lens was small and so was the homodyne signal. At very low light-intensity levels, the thermal noise and shot noise that cause the dark current become competitive for a p-i-n diode. We chose to use an avalanche photodiode in order to overcome this problem with $\mathrm{p}-\mathrm{i}-\mathrm{n}$ diodes.

Autocorrelation functions were computed by a Brookhaven Instruments analog input BI-8050 correlator after signal conditioning by an EG\&G 5113 bandpass filter amplifier. We observed experimentally that optical homodyne operation could be achieved when the polarization of both the scattered signal and the local-oscillator signal were properly matched. Optimization was achieved when one end of the graded-index lens was slowly rotated on the spectrometer, that is, the polarization of the scattered 


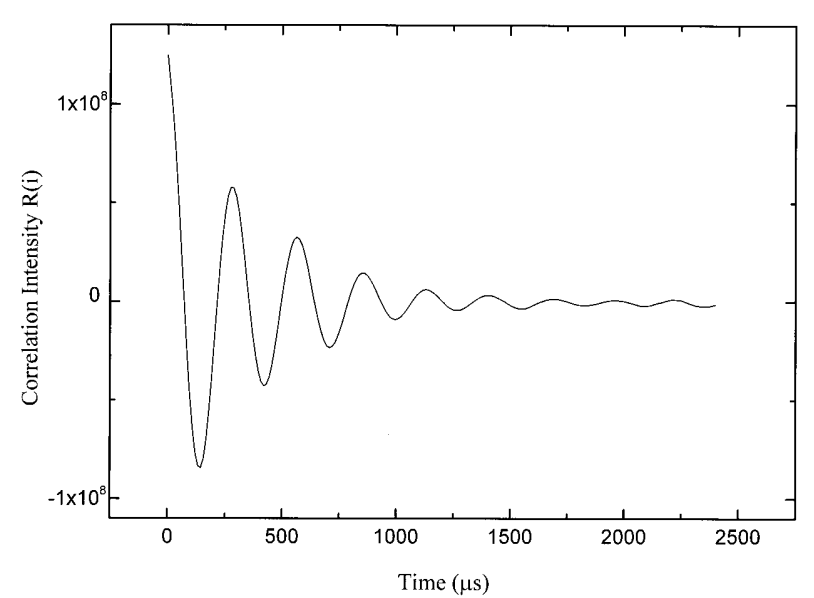

Fig. 2. Autocorrelation function data of an acetone-vapor interface taken with FOSLSS.

light mixing with the local oscillator was adjusted for optimal mixing. The coherence length requirement on this type of spectroscopy is very moderate. Based on the verifications made on the bulk optics system ${ }^{2}$ and applying this to the fiber-optics system, our calculations showed that, for this setup, the coherence length requirement is $\sim 2 \mu \mathrm{m}$ whereas the laser that we used for this experiment had a coherence length of $1 \mathrm{~cm}$.

\section{Results and Discussion}

Repeated measurements have been made on simple liquid-vapor interfaces with the above fiber-optics spectrometer at room temperature. These include air-water, air-ethanol, and air-acetone interfaces. Initial measurements were taken on a known sample, such as ethanol, to obtain the scattering angle and the scattered $\mathbf{k}$ vector. Once the $\mathbf{k}$ value was determined in this manner, that scattering angle is used for that particular data-acquisition run on several different samples.

Figure 2 shows the autocorrelation spectrum for an acetone-vapor interface observed with our FOSLSS system. This signal corresponds to the correlation function $R(\tau)$, where,

$$
R(\tau)=A+B \cos \left(\omega_{0} \tau\right) \mathrm{e}^{(-\Gamma \tau)}
$$

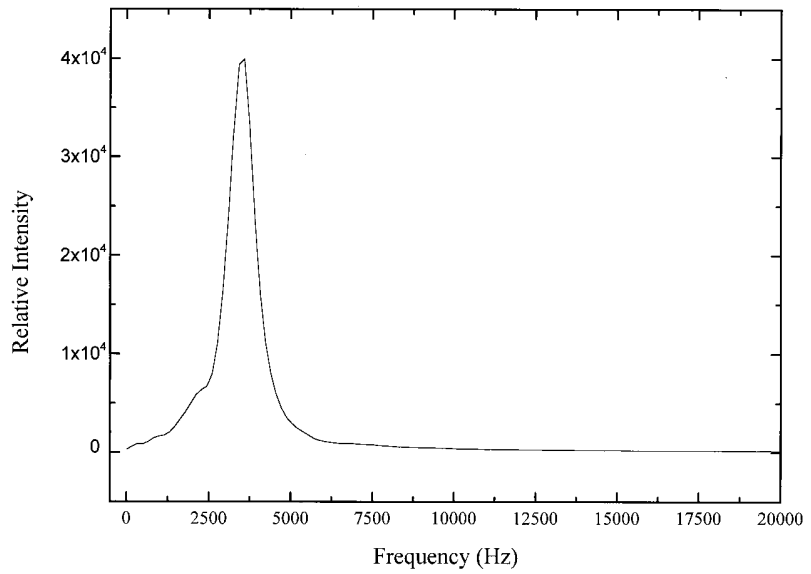

Fig. 3. Power spectrum of acetone-vapor interface taken with FOSLSS. The peak is near $3.75 \mathrm{KHz}$, which corresponds to a $\mathbf{k}$ vector of $265 \mathrm{~cm}^{-1}$.

Apart from the constants $A$ and $B, \omega_{0}$ and $\Gamma$ are the center frequency and half-width at half-maximum of a Lorentzian,

$$
S(\omega)=a_{0}\left[\frac{\Gamma}{\Gamma^{2}+\left(\omega-\omega_{0}\right)^{2}}+\frac{\Gamma}{\Gamma^{2}+\left(\omega+\omega_{0}\right)^{2}}\right],
$$

which pertains to the frequency spectrum obtained from the Fourier transformation of the correlation function $R(\tau)$. Figure 3 shows the corresponding power spectrum for the acetone-vapor interface, and the $\mathbf{k}$ vector for this particular set of experiments is $265 \mathrm{~cm}^{-1}$. The dispersion relation relates the kinematic viscosity $v$ and the surface tension $\gamma$ to $\omega_{0}$ and $\Gamma$ with the associated wave vector $\mathbf{k}$ in the first-order approximation as

$$
\omega_{0}(\mathbf{k})=\sqrt{\gamma / \rho}|\mathbf{k}|^{3 / 2} \text { and } \Gamma=2|\mathbf{k}|^{2}(\mu / \rho) .
$$

Here $\rho$ is the density of the liquid and $\mu$ is the viscosity. The data-reduction code developed by Edwards et al. ${ }^{4}$ and Mann ${ }^{9}$ has been used for analyzing the results presented here.

Results obtained with our fiber spectrometer have been compared with the established values from literature. Our measurement of the surface tension value gives a deviation of less than $1 \%$ from the established results and approximately a $5 \%$ devia-

\begin{tabular}{|c|c|c|c|c|}
\hline Liquid & $\begin{array}{l}\text { Wave Vector } \\
\mathbf{k}\left(\mathrm{cm}^{-1}\right)\end{array}$ & \multicolumn{2}{|c|}{$\begin{array}{c}\text { Measured and Calculated from Dispersion } \\
\text { Equation }\end{array}$} & $\begin{array}{l}\text { Established } \\
\text { Values }\end{array}$ \\
\hline \multirow[t]{2}{*}{ Ethanol } & 264 & Surface tension $(\mathrm{mN} / \mathrm{m})$ & 22.76 & 22.75 \\
\hline & & Viscosity $(\mathrm{cP})$ & 1.186 & 1.19 \\
\hline \multirow[t]{2}{*}{ Acetone } & 256 & Surface tension $(\mathrm{mN} / \mathrm{m})$ & 23.00 & 23.04 \\
\hline & & Viscosity $(\mathrm{cP})$ & 0.326 & 0.3069 \\
\hline \multirow[t]{2}{*}{ Water } & 266 & Surface tension $(\mathrm{mN} / \mathrm{m})$ & 71.87 & 71.98 \\
\hline & & Viscosity $(\mathrm{cP})$ & 0.94 & 0.89 \\
\hline
\end{tabular}
tion for viscosity. Our results are given in Table 1,

Table 1. Summary of Interfacial Tension and Viscosity Data on Simple Liquid-vapor interfaces measured by FOSLSS 
in which they are compared with established values.

Additionally, in the data-reduction process, comparison of the measured signal-to-noise ratio of the homodyne signal of the FOSLSS with the data we have taken with bulk optics systems shows that our signal-to-noise ratio is approximately three times higher than that obtained with a conventional bulk optics grating design. The local-oscillator strength on a bulk optics system depends on the first-order intensity of the grating and it is fixed, whereas with the variable-ratio coupler one can conveniently adjust the local-oscillator strength to attain the best signal-to-noise ratio on the homodyne beating.

Because the amount of light scattered back from the liquid-vapor interface is extremely small, working with conventional optics systems become difficult at high $\mathbf{k}$ vectors, as discussed by K. Sakai et al. ${ }^{6}$ Their measurements extended to a $\mathbf{k}$ vector of 3000 $\mathrm{cm}^{-1}$ (near $6 \mathrm{MHz}$ in frequency) after improvements were made both in the optical design setup and in the signal processing electronically. With our fiberoptics surface-light-scattering system, as an alternative approach for extending to higher $\mathbf{k}$ vectors, we can enhance this weak signal by optically amplifying it before homodyne mixing it with the local-oscillator light. This also bypasses the problem of amplifying the associated electronic noise when amplifying the signal electronically. Currently we are investigating this system by using one type of rare-earth-doped optical amplifier to enhance the weakly scattered signal optically. The scattered signal is fed into the input section of the optical amplifier where the signal is amplified in the active fiber section, and the amplified signal is then combined with the localoscillator light entering into the variable-ratio coupler.

This new FOSLSS system has several advantages such as design flexibility, an improved signal-to-noise ratio, compactness, minimal weight, and low power consumption. It also makes it possible to design a system that can scan in wave-number space continuously. That is to say a set of correlation functions can be collected, each with a different $\mathbf{k}$ value set under computer control. In contrast, a grating system requires a set of gratings or an optical system in which the magnification can be varied continuously and accurately.

The Microgravity Research Division of NASA supported this research.

\section{References}

1. D. Langevin, Light Scattering by Liquid Surfaces and Complementary Techniques, Vol. 41 of the Surface Science Series (Marcel Dekker, New York, 1992), Part 1, Chaps. 1 and 3.

2. S. Härd, Y. Hamnerius, and O. Nilsson, "Laser heterodyne apparatus for measurements of liquid surface properties-theory and experiments," J. Appl. Phys. 47, 2433-2442 (1976).

3. L. Lading, J. A. Mann Jr., and R. V. Edwards, "Analysis of a surface light scattering spectrometer," J. Opt. Soc. Am. A 6, 1692-1701 (1989).

4. R. V. Edwards, R. S. Sirohi, J. A. Mann Jr., L. B. Shih, and L. Lading, "Surface fluctuation scattering using grating heterodyne spectroscopy," Appl. Opt. 21, 3555-3568 (1982).

5. E. Mazur and S. Chung, "Light scattering from liquid-vapor interface," Physica 147A, 387-406 (1987).

6. K. Sakai, P. K. Choi, H. Tanaka, and K. Takagi, "A new light scattering technique for a wide-band ripplon spectroscopy at the MHz region," Rev. Sci. Instrum. 62, 1192-1195 (1991).

7. T. M. Jorgensen, "Information processing in optical measuring systems," Ph.D. dissertation, RISØ-R-645(EN) (Risø National Laboratory, Roskilde, Denmark, 1992).

8. W. V. Meyer, J. A. Lock, H. M. Cheung, T. W. Taylor, P. Tin, and J. A. Mann, Jr., "Hybrid reflection-transmission surface lightscattering instrument with reduced sensitivity to surface sloshing," Appl. Opt. 36, 7605-7614 (1997).

9. J. A. Mann Jr., "Surface light scattering spectroscopy," in Light Scattering and Photon Correlation Spectroscopy, E. R. Pike and J. B. Abbiss, eds., NATO ASI Series (Kluwer, Dordrecht, The Netherlands, 1997). 